\title{
Propiedades psicométricas de la Escala de Dependencia al Ejercicio-Revisada (EDS-R) en usuarios colombianos de gimnasios Psychometric properties of the Exercise Dependence Scale -Revised (EDS-R) in Colombian gyms users

\author{
* J orge Emiro Restrepo, **Tatiana Castañeda Q uirama, ***Gina Paula Cuartas M ontoya \\ *Tecnológico deAntioquia(Colombia), **Politécnico Grancolombiano (Colombia) ***Univer sidad Cooperativa de Colombia
}

(Colombia)

\begin{abstract}
Resumen: el artículo presentaun estudio psicométrico de laEscalade Dependenciaal Ejercicio Físico - Revisada(EDS-R) en usuarios de gimnasios colombianos con el objetivo de analizar su estructura factorial, consistencia interna, confiabilidad de constructo y su validez convergente, discriminantey de criterio. Se realizó una investigación instrumental en laque participaron 515 usuarios de gimnasios (44.3\% hombresy $55.7 \%$ mujeres) con edadesentrelos 18 y $\operatorname{los} 58$ años ( $M d=25 ; R I Q=21-30)$ delaciudad de M edellín. Seanalizólaversión en español (Sicilia\& González-Cutre, 2011) de laEscalade D ependenciaal Ejercicio Físico - Revisada(EDS-R) (D owns, H ausenblas \& Nigg, 2004). Se ejecutaron análisisfactoriales confirmatorios y exploratorios. Resultados: laestructuraoriginal de laEDS-R mostró unaadecuada consistenciainternay validez factorial, confirmadaatravés de losíndices debondad deajusteparael modelo de siete dimensiones. El alfade Cronbach fue de. 917 y el coeficiente de dosmitades de Guttman fue de.924. Losíndices de bondad de ajuste paralaversión original fueron: PCMIN $/$ DF = 2.291; RMR =.091; GFI = .917; $\mathrm{AGFI}=.886 ; \mathrm{CFI}=.951 ; \mathrm{RMSEA}=.056(90 \% \mathrm{Cl}=.049-.063) ; \mathrm{PCLOSE}=.093$. Sin embargo, losvaloresdelavarianzamediaextráda, lamáximavarianza compartiday lafiabilidad máxima pusieron en evidenciadificultades con la confiabilidad de constructo, la validez convergente y validez discriminante. Tampoco hubo buenos resultadosal analizar lavalidez de criterio. Para analizar si los datos en la muestra se ajustaban mejor a una estructura factorial diferente, se realizó un análisis factorial exploratorio complementario que llegó a un modelo de cinco factores, con los mismos 21 ítems. Sin embargo, laevaluación dela fiabilidad compuesta, validez convergentey validez discriminante arrojó mejores resultadosqueel modelo de sietefactores.

Palabrasclave: validez, confiabilidad, medición, dependenciaal ejercicio físico.
\end{abstract}

Abstract:Thearticle presentsapsychometric study of thePhysical Exercise DependenceScale-Revised (EDS-R) in Colombian gym users with the aim of anal yzing itsfactorial structure, internal consistency, construct reliability and its convergent, discriminant and criterion validity. An instrumental research was carried out with the participation of 515 gym users ( $44.3 \%$ men and $55.7 \%$ women) aged between 18 and 58 years ( $\mathrm{M} \mathrm{d}=25 ; \mathrm{RIQ}=21-30$ ) from the city of M edellín. The Spanish version (Sicilia\& G onzález-Cutre, 2011) of the Physical Exercise Dependence Scale-Revised (EDS-R) (D owns, H ausenblas\& N igg, 2004) was analyzed. Confirmatory and exploratory factor analyses were performed. Results: The original structure of theEDS-R showed adequate internal consistency and factorial validity, confirmed through the goodness-of-fit indicesfor the seven-dimensional model. Cronbach's al pha was .917 and the Guttman two-half coefficient was. 924. The goodness-of-fit indices for the original version were: PCM IN / DF = 2.291; RMR $=.091 ; \mathrm{GFI}=.917 ; \mathrm{AGFI}=.886 ; \mathrm{CFI}=.951 ; \mathrm{RMSEA}$ $=.056(90 \% \mathrm{Cl}=.049-.063) ; \mathrm{PCLOSE}=.093$. However, the valuesfor mean varianceextracted, maximum shared variance and maximum reliability reveal ed difficulties with construct reliability, convergent validity and discriminant validity. Therewere also no-good results when analyzing criterion validity.To analyze whether the datain the sample fit better to adifferent factor structure, acomplementary exploratory factor analysiswas performed that arrived at a five-factor model, with the same 21 items. However, the assessment of composite reliability, convergent validity and discriminant validity yielded better resultsthan the seven-factor model.

Key words: validity, reliability, measurement, dependence on physical exercise.

\section{Introducción}

Las adicciones conductuales son un campo de estudios en desarrollo. En la actualidad, solo el juego patológico está incluido en el DSM-5 como un trastorno no relacionado con sustancias dentro de lacategoríadeTrastornos relacionados con sustancias y trastornosadictivos. Aunque no están incluidos en esta clasificación, diversos comportamientos compulsivos tienen características

Fecha recepción: 23-11-20. Fecha de aceptación: 18-02-21

Jorge Emiro Restrepo

jorge. restrepo67@ tdea.edu.co psicopatológicas muy similares a las de las adicciones a sustancias, como la adicción a internet, la adicción al sexo, la adicción a los video juegos, entre otras (Grant, Potenza, Weinstein \& Gorelick, 2010).

Practicar ejercicio frecuentemente es en la actualidad, y en la cultura occidental, un comportamiento común. En condiciones normales, es una actividad saluda ble y se llegó a considerar como una adicción positiva (Glasser, 1976). Sin embargo, en al gunos casos esta práctica puede llegar a ser excesiva y descontrolada y puede, incluso, desencadenar síntomas comportamentales, cognitivos y emocionales propios de una adicción tal y como está conceptualizada en el DSM -5. Cuando esto 
ocurre, se presenta un fenómeno que ha recibido varias denominaciones: ejercicio obligatorio (Pasman \& Thompson, 1988), abuso del ejercicio (Davis, 2000), ejercicio compulsivo (Lichtenstein, Hinze, Emborg, Thomsen \& Hemmingsen, 2017), adicción al ejercicio físico (Berczik et al., 2012) y dependencia al ejercicio físico (Hausenblas \& Downs, 2002a). Las personas que se ejercitan frecuentemente, como los deportistas, los usuarios de gimnasios o centros deportivos son una población de interés (Sancho, Ruiz-J uan \& Arufe, 2018)

Seha sugerido queladenominación «adicción al ejercicio físico» es la más acertada puesto que incluye tanto la compulsión como la dependencia, aunque la denominación «dependencia al ejercicio físico» parecería ser la de uso más frecuente (Berczik et al. , 2012). Cual quiera que sea el término, esta conducta excesiva se caracteriza por ser un patrón no adaptativo que genera malestar psicológico y social. Por ser una adicción, se entiende como un comportamiento que resulta placentero o puede reducir la ansiedad, el estrés 0 algún otro malestar psicológico, y se caracteriza por la incapacidad de ser controlado y su persistencia pese a los efectos negativos (Goodman, 1990).

Existen varios instrumentos de medición para este tipo de adicción. En un inicio se desarrollaron escalas unidimensionales, como la Escala de Compromiso a Correr «Commitment to Running Scale» (Carmack \& Martens, 1979) y la «N egativeAddiction Scale» (Hailey \& Bailey, 1982). La primera fue validada en español en un total de 174 corredores españoles (hombres y mujeres) que participaron en una maratón (Ruiz-Juan \& Zarauz, 2011). La segunda no tiene adaptación al espa ñol. El Cuestionario de Ejercicio Obligatorio «O bligatory Exercise Q uestionnaire» (Pasman \& Thompson, 1988), construido posteriormente, tampoco dispone de validación o adaptación al español. Finalmente, la Escala de Adicción a Correr «Running Addiction Scale» (Chapman \& De Castro, 1990), que fue validada y adaptada al español utilizando la misma muestra de 174 corredores españoles de maratón (Zarauz \& Ruiz-Juan, 2011).

Uno de los primeros instrumentos multidimensionales que se construyó fue el Cuestiona rio de Dependencia al Ejercicio «Exercise Dependence Q uestionnaire» (O gden, Veale, \& Summers, 1997), con ocho factores: interferencia en la vida social/ familiar/ trabajo, recompensa positiva, síntomas de abstinencia, ejercicio para controlar el peso, comprensión del problema, ejercicio por razones sociales, ejercicio por ra zones de salud y comportamiento estereotipado. Este instrumento fue elaborado a partir de una muestra de 449 hombres y mujeres que practicaban ejercicio físico por más de cuatro horas a la semana. Luego se creó el Inventario de Adicción al Ejercicio «Exercise Addiction Inventory» (Terry, Szabo, \& Griffiths, 2004), a partir de una muestra de 200 hombres y mujeres. La mitad de ellos eran estudiantes de ciencias del deporte que participan en actividades deportivas. Este instrumento se desarrolló con una estructura de seis factores, que correspondían a los componentes de la adicción al comportamiento establecidos por Griffiths (1996): sal iencia, modificación del estado de ánimo, tolerancia, síntomas de abstinencia, conflicto y recaída. Este inventario fue adaptado y validado en 584 estudiantes universitarios españoles (437 estudiantes de educación física y deportes) (Sicilia, Alías-García, Ferriz \& Moreno-M urcia, 2013).

Utilizando los criterios del DSM -IV para la dependencia de sustancias, Hausenblas y D owns (2002b) crea ron laEscaladeD ependenciaal Ejercicio Físico «Exercise Dependence Scale» operacionalizando multidimensional mentela dependencia como un patrón inadaptado de ejercicio que produce alteraciones clínicamente significativas y se manifiesta en por 10 menos tres de los siguientes criterios. 1) Tolerancia: la necesidad de practicar más actividad física para lograr los efectos deseados, o una disminución de los efectos ante la misma cantidad de actividad realizada. 2) Abstinencia: síntomas característicos de abstinencia del ejercicio (ansiedad, fatiga, agotamiento), o la realización de actividad para evitar estos síntomas. 3) Efectos desea dos: la actividad física se realiza durante más tiempo 0 en mayor cantidad de lo inicialmente deseado. 4) Falta de control: deseo persistente o un esfuerzo infructuoso de controlar o interrumpir la actividad física. 5) Tiempo: se invierte mucho tiempo en actividades relacionadas con la actividad física. 6) Reducción de otras actividades: las actividades sociales, laborales y recreativas se disminuyen debido alaactividad física. 7) Continuación: se realiza la actividad física a pesar de tener problemas psicológicos o físicos persistentes causados o agravados por la misma actividad.

Para evaluar estos criterios, crearon un conjunto inicial de 35 ítems basado en entrevistas con personas que hacían ejercicio y una revisión de los instrumentos de medición existentes en el área. Cuatro expertos en ciencias del ejercicio y cuatro ejercitantes interesados revisaron la adecuación, legibilidad y comprensión para establecer lavalidez de contenido (Hausenblas \& D owns, 2002b). Luego de esta revisión se eliminaron 6 ítems. 
Al realizar el primer análisis de confiabilidad, detecta ron que dos ítems tenían muy baja discriminación. La consistencia interna reportada para esta versión final de 27 ítems fue de un alfa de Cronbach de 0.93. En un segundo estudio dentro de la misma publicación, aña dieron un ítem asociado con el criterio de Abstinencia. Esta versión de 28 ítems fue la definitiva y se aplicó a 862 estudiantes universitarios. Tuvo un alfa de 0.94 , y adecuada validez discriminante y validez concurrente.

Posteriormente, Downs, Hausenblas \& Nigg (2004) analizaron las propiedades psicométricas de una versión revisada: la Escala de Dependencia al Ejercicio Físico Revisada (EDS-R) «Exercise Dependence ScaleRevised» en una muestra de 408 estudiantes universitarios que participaban en una clase de fitness y se ejercitaban, como mínimo, tres horas por semana. Específicamente, analizaron la validez factorial, la validez convergente, laconsistenciainternay la confiabilidad test-retest de la versión de 28 ítems (Hausenblas \& Downs, 2002b). Tras realizar los análisis y ajustar el modelo de medida para mejorar los índices de bondad de ajuste (eliminando los ítems con cargar factoriales inferiores a 0.5 y los ítems complejos identificados a través de la prueba de multiplicadores de Lagrange), el modelo demedida final tuvo siete factores con tresítems cada uno para un total de 21 ítems. Los valores de los índices de bondad de ajuste fueron: $T L I=.95 ; C F I=$ $.96 ;$ RMSEA $=.06 ;$ AASR $=.03 ; p<.05$. Adicionalmente, reportaron que la consistencia interna, la varianza media extraída y la confiabilidad, a tra vés del 0 mega de $M$ acD onald, fueron adecuados, y la confiabilidad test-retest fue excelente, con la excepción de la subescala de Reducción de 0 trasActividades. Utilizando las frecuencias de ejercicio físico reportadas por los participantes, lograron establecer una adecuada va lidez convergente del instrumento, pues pudo establecer que el grupo de riesgo para dependencia era el de más frecuencia de ejercicio.

Esta versión de siete factores y 21 ítems ha sido analizada en diferentes países con personas que se ejercitan frecuentemente. La tabla 1 presenta al gunos de estos estudios y sus principales hallazgos. Estas adaptaciones, validaciones y análisis de las propiedades psicométricas se han realizado únicamente en Europa. En Latinoamérica no se ha hecho ningún estudio de este tipo con esta escala en particular. Para continuar avanzando en la estandarización y adaptación de instrumentos de medición en esta región, esta investigación tuvo como objetivo analizar las propiedades psicométricas de la EDS-R en usuarios de gimnasios colombianos.
Específicamente, se examinó la estructura factorial, la consistencia interna y la validez del criterio analizando las diferencias en los síntomas de la dependencia según la frecuencia de ejercicio.

Para tal propósito, se utilizó la versión en español validada por Sicilia \& González-Cutre (2011) con usuaz rios de centro deportivos en España. Esta versión demostró coincidir con la versión original de siete factores y 21 ítemes, adecuados valores de consistencia interna de las escalas (con excepción de Reducción de otras actividades), adecuada estabilidad temporal y va lidez de criterio. Considerando la revisión de literatu$\mathrm{ra}$, suponemos que es posible que la estrucutra factorial no se ajuste al modelo original de siete factores. Los estudios previos no han concidio siempre en ese número de factores. Respecto a la val idez convergente y discriminante, la hipótesis es que podrían presentarse dificultades si el modelo de siete factores no se corresponde con los datos. En relación con la validez de criterio, se espera que el instrumento sí diferencie entre los grupos en función de la frecuencia de ejercicio.

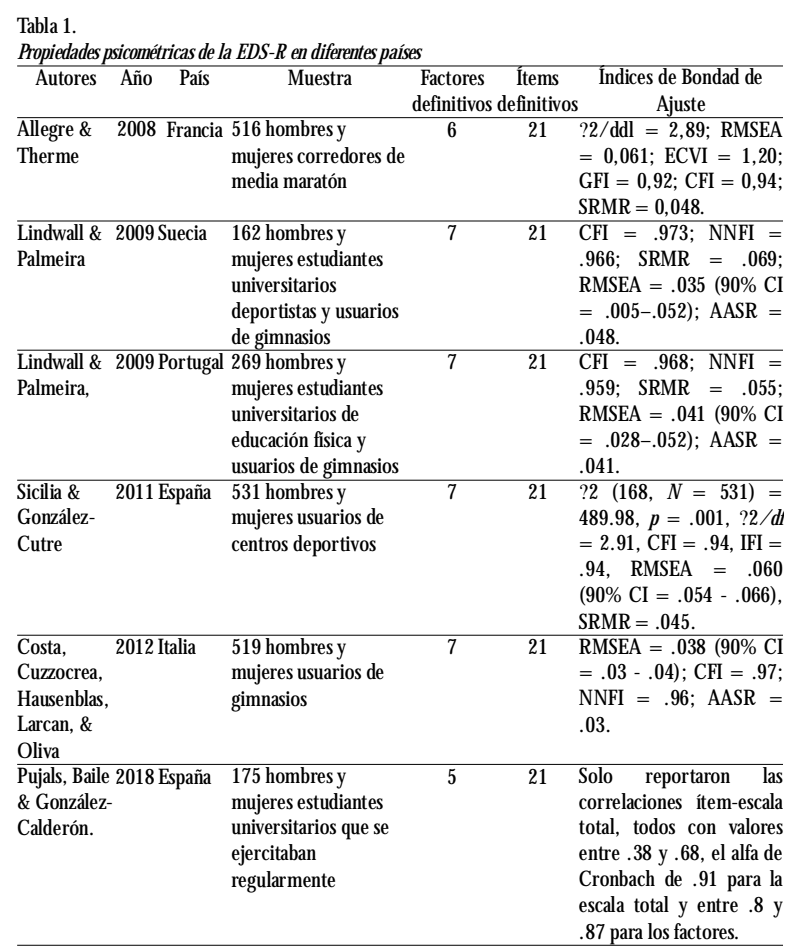

\section{Material y método}

\section{D iseño}

Se realizó una investigación instrumental con el objetivo de examinar la estructura factorial, la consistenciainterna, la confiabilidad de constructo, lavalidez convergente, discriminante y de criterio de la versión en español (Sicilia \& González-Cutre, 2011) de la Escala 
de Dependencia al Ejercicio Físico - Revisada (EDS-R) (Downs, Hausenblas \& Nigg, 2004).

\section{Instrumento}

Escala de Dependencia al Ejercicio Físico - Revisada (EDS-R) (D owns, Hausenblas \& Nigg, 2004; versión en español validadapor Sicilia\& González-Cutre, 2011). Es un instrumento diseñado para evaluar la dependencia al ejercicio físico a través de 21 ítems con respuesta en una escala Likert de 6 puntos, que va de 1 (nunca) a 6 (siempre). Los ítems están agrupados en siete factores: abstinencia (ítems 1, 8 y 15), continuación (ítems 2,9 y 16), tolerancia (ítems 3,10 y 17), falta de control (ítems 4,11 y 18), reducción de otras actividades ('items 5, 12 y 19), tiempo (ítems 6,13 y 20), y efectos desea dos (ítems 7, 14 y 21). La EDS-R permite clasificar a los individuos en tres grupos: con riesgo de dependencia del ejercicio (puntuaciones Likert de 5-6 en al menos tres de los siete criterios), sintomáticos no dependientes (puntuaciones Likert de 3-4 en al menos tres criterios, o puntuaciones de 5-6 combinadas con puntuaciones de 3-4 en tres criterios, pero sin cumplir las condiciones de la sintomatología no dependiente), y asintomáticos no dependientes (puntuaciones Likert de 1-2 en al menos tres criterios, sin cumplir las condiciones de la sintomatología no dependiente).

\section{Participantes}

En el estudio participaron 515 usuarios de gimna sios (228 hombres y 287 mujeres) con edades entre los 18 y los 58 años ( $M d=25 ; \mathrm{RIQ}=21-30$ ) de la ciudad de Medellín. Solo se incluyeron mayores de edad que llevaran más de tres meses asistiendo al gimnasio regularmente. El $63.7 \%$ de los participantes estaba en el estrato socioeconómico medio (estratos 3 y 4). El 47,2 $\%$ reportó estar cursando o tener educación universita ria finalizada. El 22,9 \% informó estar cursando o tener educación técnica o tecnológica finalizada. Estás personas llevaban, en su mayoría, más de 12 meses asistiendo a los gimnasios ( $M d=24 ; R I Q=12-49$ ) y asistían entre 4 y 5 días a la semana ( $M d=5 ; R I Q=4-5)$. Su rutina de ejercicio físico era de dos horas por día ( $M d=$ $2 ; \mathrm{RIQ}=1-2$.

\section{Procedimiento}

Los participantes se contactaron directamente en gimnasios de la ciudad de Medellín, Departamento de Antioquia, en donde practicaban diversos tipos de actividades físicas, como crossit, entrenamiento funcional, levantamiento de pesas y empleo tradicional de máqui- nas. Se les explicó el objetivo de la investigación y se les solicitó que leyeran y firmaran el consentimiento informado, que fue previamente aprobado por un comité de bioética. Losinstrumentosfueron diligenciadosmediante auto reporte, luego de que los participantes recibieran la información suficiente sobre cómo responderlos. Los participantes respondieron los instrumentos en sus hogares y luego los entregaron a los investigadores. Se contactaron 600 personas inicialmente. Un $14.2 \%$ no regresó los instrumentos.

\section{Normas éticas}

Se dio cumplimiento a lo establecido en la Ley 1090 de 2006 del Colegio Colombiano de Psicólogos que reglamenta el ejercicio de la profesión de la psicología y establece el correspondiente código deontológico y bioético, así como la Resolución 8430 de 1993 del Ministerio de Salud por la cual se establecen las normas científicas, técnicas y administrativas para la investigación en salud. Todos los participantes firmaron el consentimiento informado. El proyecto fue revisado y aprobado por un comité de bioética.

\section{Análisis de datos}

Los datos fueron sistematizados y analizados utilizando el programaIBM SPSS v. 24 junto con IBM AM OS v. 24. Los valores perdidos se estimaron mediante el método de imputación múltiple. Primero, se ejecutaron los análisis descriptivos para cada uno de los ítems y se calculó la correlación ítem- total en la versión original. Seguidamente se realizó el análisis de consistencia interna mediante el alfa de Cronbach y el coeficiente de dos mitades de Guttman. Luego, se ejecutó un aná lisis factorial confirmatorio (AFC) de la estructura original a través del AM OS (Byrne, 2009) para conocer el ajuste de ese modelo. Debido a que el coeficiente de Mardiafue alto (101,392), se utilizó el método de máxima probabilidad con el procedimiento de bootstrapping. También se analizó la validez de este modelo de medida original a través de la Fiabilidad compuesta (CR), la Varianza media extraída (AVE), la Máxima varianza compartida (MSV), y la Fiabilidad máxima (H) $(\operatorname{MaxR}(H))$. Posteriormente, se ejecutó un Análisis Factorial Exploratorio (AFE) con los 21 ítems para encontrar un modelo de medida con mejores indicadores de fiabilidad compuesta, validez convergente y validez discriminante. El AFE se ejecutó utilizando el método de Factorización de ejes principales y rotación Promax con normalización Kaiser. Finalmente, se examinó la validez del criterio analizando las diferencias en los sín- 
tomas de la dependencia según la frecuencia de ejercicio. EsteAFE se realizó con el propósito de conocer cuál era el mejor modelo factorial para los datos en esta muestra y proporcionar información para futuros estudios que permitan continuar avanzando en la estandarización y adaptación de instrumentos de medición en esta región.

\section{Resultados}

En laTabla 2 se presentan las medidas de resumen de todos los items, así como sus valores de asimetría y curtosis. De acuerdo con estos últimos, no existe normalidad en la distribución de la mayoría de los items. Respecto a la correlación item-total, los valores estuvieron entre .5 y .7 y pueden considerarse adecuados. El alfa de Cronbach fue de .917 y el coeficiente de dos mitades de Guttman fue de.924. Losíndices de bondad de ajuste para la versión original (Figura 1) fueron: PCMIN/ DF $=2.291 ; \mathrm{RMR}=.091 ; \mathrm{GFI}=.917 ; \mathrm{AGFI}$ $=.886 ; \mathrm{CFI}=.951 ; \mathrm{RMSEA}=.056(90 \% \mathrm{Cl}=.049$ .063); PCLOSE $=.093$. Los coeficientes de regresión, las varianzas y covarianzas fueron todos estadísticamente significativas $(p<.001)$. Aunque los indicadores de ajuste del modelo fueron satisfactorios, las medidas de validez (Tabla 3) mostraron dificultades en cuanto a la fiabilidad compuesta, la validez convergente y la validez discriminante, en los factores Falta de control, Reducción de otras actividades y Efectos deseados. Estos fallos se deben probablemente a valores muy altos que sugieren problemas de colinealidad o redundancia.

\begin{tabular}{|c|c|c|c|c|c|}
\hline & Media & $\mathrm{DE}$ & Asimetría & Curtosis & Correlación Item-total \\
\hline Item1 & 3.58 & 1.68 & -.23 & -1.14 & $.52^{*}$ \\
\hline Item2 & 2.62 & 1.68 & .66 & -.85 & $.50 *$ \\
\hline Item3 & 4.46 & 1.43 & -.85 & -.02 & $.60 *$ \\
\hline Item4 & 3.15 & 1.60 & .13 & -1.07 & $.57 *$ \\
\hline Item5 & 2.29 & 1.39 & .89 & -.06 & $.57 *$ \\
\hline Item6 & 3.35 & 1.53 & 15 & -.97 & $.70^{*}$ \\
\hline Item7 & 2.29 & 1.33 & .92 & .09 & $.62^{*}$ \\
\hline Item8 & 3.17 & 1.77 & .14 & -1.36 & $.62^{*}$ \\
\hline Item9 & 2.03 & 1.43 & 1.25 & .48 & $.52^{*}$ \\
\hline Item10 & 4.19 & 1.50 & -.51 & -.69 & $.64 *$ \\
\hline Item11 & 3.12 & 1.55 & .17 & -.99 & $.61^{*}$ \\
\hline Item12 & 2.38 & 1.48 & .83 & -.36 & $.61^{*}$ \\
\hline Item13 & 3.37 & 1.57 & .08 & -1.03 & $.68^{*}$ \\
\hline Item14 & 2.62 & 1.41 & .59 & -.55 & $.70 *$ \\
\hline Item15 & 3.64 & 1.64 & -.20 & -1.15 & $.61^{*}$ \\
\hline Item16 & 2.32 & 1.54 & .94 & -.26 & $.55^{*}$ \\
\hline Item17 & 3.69 & 1.62 & -.18 & -1.13 & $.66^{*}$ \\
\hline Item18 & 2.90 & 1.50 & .38 & -.79 & $.61^{*}$ \\
\hline Item19 & 1.74 & 1.17 & 1.62 & 1.92 & $.50 *$ \\
\hline Item20 & 3.20 & 1.51 & .19 & -.91 & $.73 *$ \\
\hline Item21 & 2.71 & 1.44 & .48 & -.66 & $.72 *$ \\
\hline
\end{tabular}

El Análisis Factorial Exploratorio (AFE) con los 21 ítems de la versión original encontró cinco factores (21 ítems) que explicaban el $57.52 \%$ de la varianza. Todos los ítems tuvieron comunalidades superiores a 0.3 y

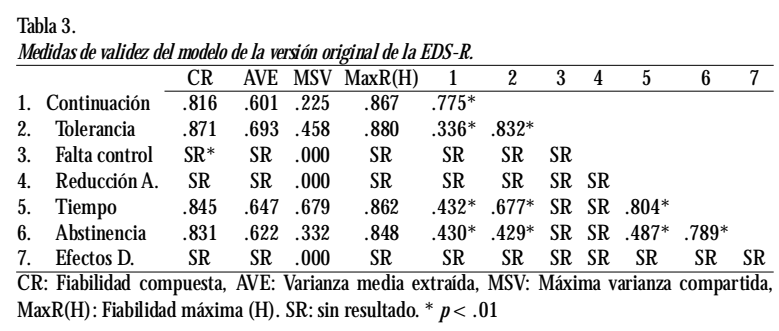

cargas factoriales entre 0.30 y 0.96 . La matriz patrón de este análisis fue exportada a AMOS a través del plugin «Pattern Matrix M odel Builder» (Gaskin, 2018). Los índices de bondad de ajuste para esta versión de cinco factores fueron: PCM IN/ DF $=3.144 ; \mathrm{RMR}=$ $.110 ; \mathrm{GFI}=.881 ; \mathrm{AGFI}=.847 ; \mathrm{CFI}=.913 ; \mathrm{RMSEA}=$ $.072(90 \% \mathrm{Cl}=.065-.079) ;$ PCLOSE $=.000$. LOS coeficientes de regresión, las varianzas y covarianzas fueron todos estadísticamente significativas ( $p<.001$ ). En laTabla 4 se presentan la Fiabilidad compuesta (CR), laVarianza media extraída (AVE), la M áxima varianza compartida (MSV), y la Fiabilidad máxima $(H)$ $(\operatorname{MaxR}(H))$ de este modelo.

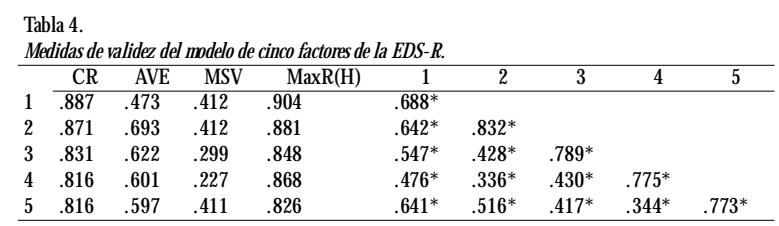

Las medidas de validez de este modelo de cinco factores son mejores que las del modelo original de siete factores. El único indicador que está por fuera de los valores estandarizados es la varianza media extraída del factor 1 (inferior a .5). En este modelo, los factores Reducción de otras Actividades, Tiempo y Efectos deseados quedaron agrupados dentro de un mismo factor, lo que no tiene justificación teórica, dada la naturaleza de los ítems que los componen.

Para anal izar las posibles diferencias de la EDS$R$ en función de la frecuencia de ejercicio físico (Tabla 5), se utilizaron dos grupos, considerando la asistencia al gimnasio durante uno o tres días por semana como una frecuencia mediabaja ( $37.1 \%$ de los usuarios), y entre cuatro y siete días, como un nivel alto $(62,9 \%$ ) (Haskell et al., 2007). Adicionalmente, se examinaron las correlaciones entre los factores y la puntuación total de la

\begin{tabular}{|c|c|c|c|c|c|c|}
\hline \multirow[t]{2}{*}{ thenton } & \multicolumn{2}{|c|}{3 días o menos por semana } & \multicolumn{2}{|c|}{4 a 7 días por semana } & \multirow[b]{2}{*}{$\mathrm{F}$} & \multirow[b]{2}{*}{$p$} \\
\hline & M & $\mathrm{DE}$ & M & $\mathrm{DE}$ & & \\
\hline Continuación & 2.31 & 1.47 & 2.32 & 1.27 & 1.481 & .96 \\
\hline Tolerancia & 3.95 & 1.46 & 4.15 & 1.32 & 1.940 & .22 \\
\hline Fal ta control & 2.99 & 1.34 & 3.07 & 1.31 & .284 & .62 \\
\hline Reducción A. & 1.99 & 1.04 & 2.17 & 1.05 & .017 & .15 \\
\hline Tiempo & 3.11 & 1.25 & 3.35 & 1.35 & 1.132 & .12 \\
\hline Abstinencia & 3.34 & 1.59 & 3.49 & 1.43 & 4.253 & .36 \\
\hline Efectos D. & 2.38 & 1.23 & 2.58 & 1.20 & .051 & .17 \\
\hline Total & 60.32 & 20.65 & 63.50 & 19.31 & .241 & .17 \\
\hline
\end{tabular}


Tabla 6.

Correlaciones entre los factores y la puntuación total de la EDS-R y los reportes de frecuencia de ejercicio físico.

\begin{tabular}{|c|c|c|c|c|c|c|}
\hline & \multicolumn{2}{|c|}{ Meses } & \multicolumn{2}{|c|}{ Días } & \multicolumn{2}{|c|}{ Horas } \\
\hline & r & $p$ & $r$ & $p$ & $r$ & $p$ \\
\hline Continuación & .013 & .786 & .013 & .789 & .046 & .345 \\
\hline Tolerancia & -.004 & .935 & .040 & .416 & -.051 & .300 \\
\hline Falta control & .018 & .711 & .018 & .719 & .026 & .600 \\
\hline Reducción A. & .030 & .549 & .099 & $.043 *$ & .032 & .520 \\
\hline Tiempo & .089 & .071 & .088 & .074 & -.068 & .168 \\
\hline Abstinencia & .024 & .627 & .055 & .266 & -.100 & $.041^{*}$ \\
\hline Efectos D. & .067 & .173 & .090 & .068 & .056 & .259 \\
\hline Total & .046 & .347 & .077 & .115 & -.017 & .732 \\
\hline
\end{tabular}

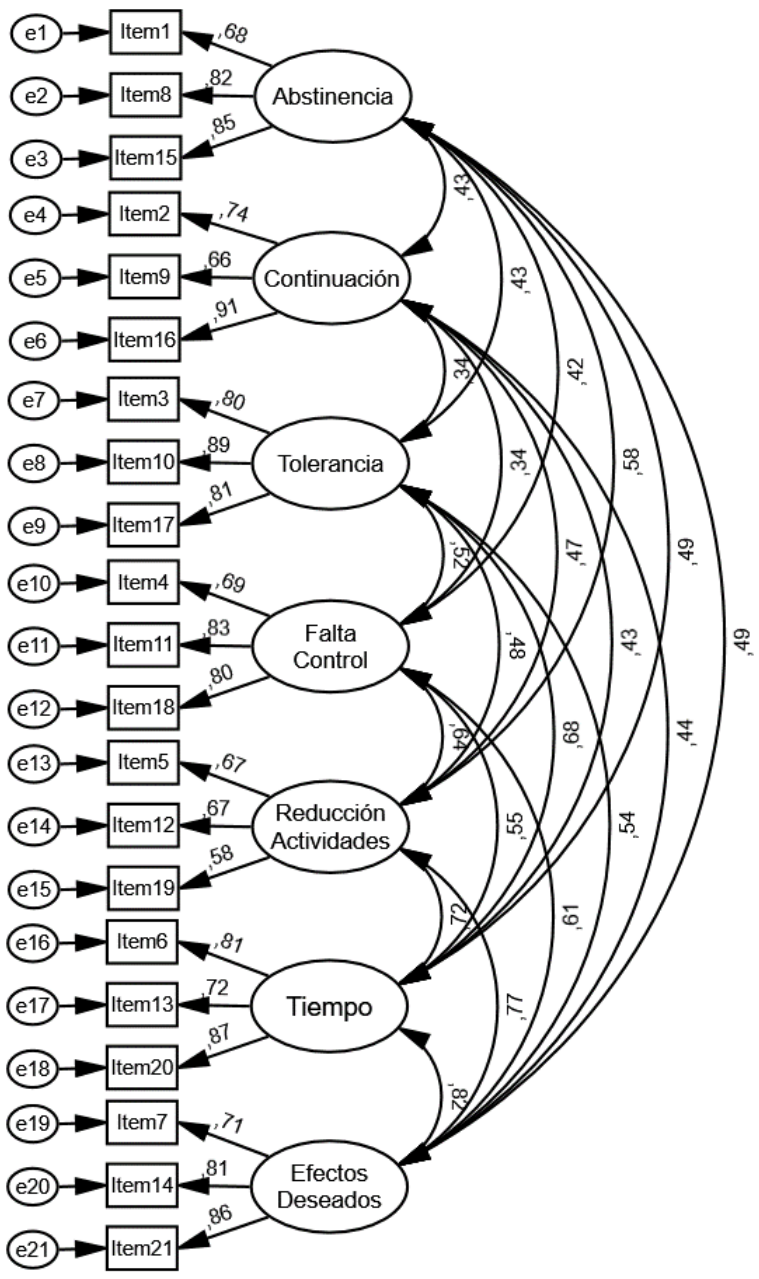

Figura 1. Análisis factorial confirmatorio de la versión original de la EDS-R

EDS-R y los reportes de frecuencia de ejercicio físico (Tabla 6).

\section{Discusión}

La estructura original de la EDS-R mostró una adecuada validez factorial, confirmada a través de los índices de bondad de ajuste para el modelo de siete dimensiones. Sin embargo, y pese a que los coeficientes de consistencia interna (Cronbach y Guttman) tuvieron valores altos, los valores de la fiabilidad compuesta no fueron adecuados y hubo problemas, especialmente con los factores de Falta de control, Reducción de otras ac- tividades y Efectos deseados, sugiriendo redundancia en los ítems, colinealidad o cualquier otra razón asociada con un fallo en la fiabilidad del constructo. Los valores de la varianza media extraída, la máxima varianza compartida y la fiabilidad máxima también pusieron en evidencia las dificultades con la val idez convergente y validez discriminante de este modelo original. Adicionalmente, los resultados de los análisis de la varianza de la dependencia al ejercicio físico por días a la semana y de los análisis de correlación entre los factores y la puntuación total de la EDS-R y los reportes de frecuencia de ejercicio físico sugirieron problemas de validez de criterio.

Al comparar algunos de los índices de bondad de ajuste de la versión de siete factores original en pobla ción colombiana con los reportados en otros países puede apreciarse un mejor ajuste del modelo colombiano, particularmente en los valores de la discrepancia mínima por grados de libertad (PCM IN/ DF $=2.291$ ) y la aproximación del error cuadrático medio (RMSEA = .056) (Kline, 1998). El índice de ajuste comparativo (CFI = .951) y el índice de bondad de ajuste (GFI = .917) tuvieron valores que indican un buen ajuste del modelo, mientras queel índice de bondad de ajuste ajustado tuvo un valor aceptable (AGFI = .886). (Bentler, 1990; Cole, 1987). Guiándose por estos resultados, puede aceptarse un buen modelo de medición de siete factores. Pero los resultados del análisis de fiabilidad compuesta, de la varianza media extraída, de la máxima varianza compartida y de la fiabilidad máxima obligaron a realizar un AFE para encontrar un modelo con mejor fiabilidad de constructo, validez convergente y validez discriminante.

El AFE a partir de la versión original llevó a un modelo de cinco factores, con losmismos 21 ítems. Aquí, los factores Reducción de otras Actividades, Tiempo y Efectos deseados quedaron agrupados dentro de un mismo factor. Los índices de bondad de ajuste de este modelo de cinco factores no fueron tan buenos como los del modelo original. Sin embargo, la evaluación de la fiabilidad compuesta (fiabilidad de constructo), validez convergente y validez discriminante arrojó mejores resultados que el modelo de siete factores. Estos resultados del modelo alternativo de cinco factores sugieren la necesidad de adaptar el instrumento ajustando su estructura factorial. Sin embargo, esta eventual modificación podría afectar la intención original de los siete criterios de Hausenblas y Downs (2002b).

De los seis estudios que analizaron la estructura factorial (Tabla 1), solo uno repor tó una estructura dife- 
rente a la original. Pujals, Baile \& González-Calderón (2018), en su análisis con 175 hombres y mujeres estudiantes universitarios españoles que se ejercitaban regularmente, encontraron una estructura de cinco factores en la que se excluían los factores de Reducción en otras actividades yT iempo. Los ítems correspondientes a estos factores fueron incluidos dentro de los factores de Tolerancia y Fal ta de control. Los resultados de ese estudio y los aquí presentados coinciden en que los factores Reducción en otras actividades y Tiempo presentan problemas en la estructura factorial del instrumento. Sin embargo, los demás estudios que analizaron la estructura factorial no hallaron tales discrepancias en la estructura factorial. No obstante, todos esos estudios se realizaron en Europa.

La estructura factorial de la versión colombiana respecto a la original podría evaluarse a través de otros métodos de extracción, como los componentes principales, los mínimos cuadrados no ponderados o la máxima verosimilitud, o modificando la técnica de rotación. También podrían realizarse el AFE depurando la muestra, controlando la edad, la escolaridad, el estrato socioeconómico 0, incluso, la frecuencia de ejercicio físico. Q uizás la heterogeneidad de los participantes afecte la estructura factorial. Una variable clave que podría estar afectando la estructura factorial es el tipo de ejercicio físico. Si bien todos los participantes eran usuarios de gimnasios, ellos practicaban diversos tipos de actividades físicas, como crossfit, entrenamiento funcional, levantamiento de pesas y empleo tradicional de máquinas.

Respecto a la validez de criterio, el análisis de la varianza de la dependencia al ejercicio físico por días a la semana no logró discriminar entre los participantes que asistían al gimnasio tres días o menos y aquellos que asistían entre cuatro y siete días. Las correlaciones entre los factores y la puntuación total de la EDS-R y los reportes de frecuencia de ejercicio físico tampoco mostraron asociación entre la dependencia y la frecuencia de ejercicio. Aunque la dependencia al ejercicio físico no se relacionanecesariamente con la frecuencia de ejercicio (mas sí con la intensidad), sí hay una tendencia en esa dirección (Costa, Hausenblas, Oliva, Cuzzocrea \& Larcan, 2013) y el instrumento debería poder diferenciar alos participantes de acuerdo con esa variable. Sicilia \& González-Cutre (2011), por ejemplo, encontraron diferencias estadísticamente significativas en todos los factoresy la puntuación total del EDS-R cuando compa raron dos grupos en función de la frecuencia de ejercicio. El grupo de mayor frecuencia de práctica física ob- tuvo puntuaciones más altas tanto en los siete factores como en el valor global de la dependencia al ejercicio, en comparación con el grupo de menor frecuencia de práctica.

Para mejorar la validez de criterio habría que recurrir a un análisis de validez de constructo. Sí la escala en su versión original aplicada a esta población no logra diferenciar a los participantes de acuerdo con su frecuencia de ejercicio habría que revisar, entonces, si el contenido de la escala, esto es, el concepto de dependencia, está adecuadamente definido. Pero este ejercicio requeriría de un análisis de la teoría desde la que se construyó el instrumento, conjuntamente con un análisis de la evidencia clínica, y esto está por fuera de los objetivos de este estudio. 0 tra forma de evaluar la va lidez de criterio sin considerar la posibilidad de que haya un fallo de contenido (constructo) del instrumento sería controlando al gunas variables que pudieran estar influyendo sobre la dependencia al ejercicio, como la insa tisfacción con la imagen corporal, la autoestima, la ansiedad, la regulación de la conducta en el deporte, los objetivos del ejercicio, entre otras (Guíu Carrera \& Leyton Román, 2019; Simón-Grima, San Martín-Salvador, Estrada-M arcén \& Casterad-Seral, 2020). Al controlar metodológicamente 0 estadísticamente algunas de estas variables sería posible determinar si el instrumento sí logra diferenciar en función de la frecuencia y así garantizar la validez de criterio. También hay que decir que no hay un criterio definitivo para distinguir cuándo es demasiado (Hausenblas y D owns, 2002a), y no puede sostenerse que «más frecuente» es «más dependiente» (Berczik et al., 2012). Así que otra posibilidad es que la frecuencia de ejercicio no sea un criterio adecuado para valorar este tipo de validez.

Este estudio, además de ser el primer análisis psicométrico de un instrumento de medición de dependencia al ejercicio físico en Colombia, tiene una ventaja sobre sus antecedentes: es el único que ha presentado valores de fiabilidad compuesta (CR), varianza media extraída (AVE), máxima varianza compartida (MSV) y fiabilidad máxima (H) (MaxR(H)). Como se dijo, estos indicadores aportan información sobre la fiabilidad de constructo, la validez convergente y la validez divergente. Los estudios preliminares se limitaban a la validez de constructo, la estructura factorial y la consistencia interna.

Estos hallazgos sobre las propiedades psicométricas de la EDS-R están lejos de ser concluyentes. Por un lado, el modelo original de siete factores presentó adecuadosíndices de bondad de ajuste y muy bueno valores 
de confiabilidad (consistencia interna), pero falló en la fiabilidad compuesta y en la validez convergente y validez discriminante. Por otro lado, el modelo hallado a través del AFE no tuvo tan buenos índices de bondad de ajuste, pero sí mejoró ostensiblemente en cuanto a la fiabilidad compuesta, la validez convergente y la validez discriminante. No hay forma de decidir sobre uno u otro modelo sin priorizar al gunos de los indicadores de validez y confiabilidad. El modelo original de siete factores de la EDS-R no cumple satisfactoriamente con ciertos indicadores de fiabilidad de constructo, validez convergente, validez divergente y validez de criterio y, por tanto, hasta donde los resultados de este estudio pueden interpretarse, su uso en población colombiana no es recomendable hasta que pueda realizarse una estandarización y adaptación o pueda tenerse un segundo estudio a través del cual puedan compararse los resultados aquí presentados.

Decir que el uso del instrumento en población colombiana no es recomendable no invalida los análisis que se hicieron. Lo que invalida es el uso del instrumento hasta que no se haya realizado un proceso de adaptación y se revise si el contenido de los ítems es adecuado y si hay items o factores que no deben incluirse (como lo demostró el AFE). Como lo demostraron los estudios de Allegre \& Therme (2008) en Francia y Pujals, Baile \& González-Calderón (2018) en España, existe la posibilidad de el modelo original de siete factores no se ajuste a ciertas poblaciones o contextos. No se hizo aquí un análisis del contenido de los ítems ni su modificación para encontrar un mejor modelo porque esto no era parte del objetivo del estudio. Sin duda, tendría que hacerse, si se desea utilizar este instrumento en la versión en español de Sicilia \& González-Cutre, (2011). Pero ese no fue el objetivo de esta investiga ción. No se pretendió adaptar la Escala de Dependencia al Ejercicio Físico - Revisada (EDS-R) en población coIombiana usuaria de gimnasios, sino presentar evidencia sobre su validez y confiabilidad y ofrecer informa ción para continuar avanzando en la estandarización y adaptación de instrumentos de medición en esta región.

\section{Referencias}

Allegre, B., \& Therme, P. (2008). Étude confirmative de l'échelle de dépendance à l'activité physique Exercise Dependence Scale-Revised pour une population francophone. L'Encéphale, 34 (5), 490495. doi:10.1016/ j.encep. 2007.08.004

Bentler, P. M. (1990). Comparative fit indexes in structural models. Psychological bulletin, 107(2), 238246. https:/ / doi.org/ 10.1037/ 00332909.107.2.238

Berczik, K., Szabó, A., Griffiths, M. D., Kurimay, T., Kun, B., U rbán, R., \& Demetrovics, Z. (2012). Exercise addiction: symptoms, diagnosis, epidemiology, and etiology. Substance U se \& Misuse, 47(4), 403-417. https:/ / doi.org/ 10.3109/ 10826084.2011 .639120

Byrne, B. M. (2009). Structural Equation Modeling With AM OS: Basic Concepts, Applications, and Programming. Taylor \& Francis.

Carmack, M. A., \& Martens, R. (1979). M easuring Commitment to Running: A Survey of Runners' Attitudesand M ental States. Journal of Sport Psychology, 1(1), 25-42. https:/ / doi.org/ 10.1123/ jsp.1.1.25

Chapman, C. L., \& De Castro, J. M. (1990). Running addiction: measurement and associated psychological characteristics. The Journal of Sports Medicine and Physical Fitness, 30 (3), 283-290.

Cole, D.A. (1987). U tility of confirmatory factor analysis in test validation research. Journal of consulting and dinical psychology, 55(4), 584-594. https:/ / doi. org/ 10.1037/ 0022-006X.55.4.584

Costa, S., Cuzzocrea, F., Hausenblas, H. A., Larcan, R., $\&$ Oliva, P. (2012). Psychometric examination and factorial validity of the Exercise Dependence ScaleRevised in Italian exercisers. Journal of Behavioral Addictions, 1(4), 186-190. https:/ / doi. org/ 10.1556/ |BA. 1.2012.009

Costa, S. , Hausenblas, H. A., O liva, P., Cuzzocrea, F., $\&$ Larcan, R. (2013). The role of age, gender, mood states and exercise frequency on exercise dependence. Journal of behavioral addictions, 2(4), 216-223. https:/ / doi.org/ 10.1556/ JBA.2.2013.014

Davis, C. (2000). Exercise abuse. International Journal of Sport Psychology, 31, 278- 289.

Downs, D. S., Hausenblas, H. A. , \& Nigg, C. R. (2004). Factorial validity and psychometric examination of the ExerciseDependenceScale-Revised. M easurement in Physical Education and Exercise Science, 8, 183-201. https:/ / doi.org/ 10.1207/ s15327841mpee0804_1 Gaskin, J. (2018). Pattern M atrix M odel Builder Plugin. Gaskination's statwiki.kolobkreations.com/

Glasser, W. (1976). Positive addiction. N ew York: Harper $\&$ Row.

Goodman, A. (1990). Addiction: Definition and implications. British Journal of Addiction, 85(11), 14031408 
Grant, J. E., Potenza, M. N., Weinstein, A., \& Gorelick, D. A. (2010). Introduction to behavioral addictions. The American Journal of Drug and Alcohol Abuse, 36(5), 233-241. https:/ / doi.org/ 10.3109/ 00952990.2010 .491884

Guíu Carrera, M., \& Leyton Román, M. (2019). Perfil psicológico en corredores de ultramaratón (Psychological profile in ultramarathon runners). Retos, 36, 310-317. https:/ / doi. org/ 10.47197/ retos. v36i36.69119

Hailey, B. J., \& Bailey, L. A. (1982). Negative addiction in runners: A quantitative approach. Journal of Sport Behavior, 5, 150-154.

Haskell, W. L., Lee, I.-M., Pate, R. R., Powell, K. E., Blair, S. N., Franklin, B. A., et al. (2007). Physical activity and public health: U pdated recommendation for adults from theAmerican College of Sports Medicine and the American Heart Association. M edicine and Science in Sports and Exercise, 39, 1423-1434. https: / / doi.org/ 10.1249/ mss.0b013e3180616b27

Hausenblas, H. A., \& Downs, D. S. (2002a). Exercise dependence: A systematic review. Psychology of Sport and Exercise, 3(2), 89- 123. https:/ / doi. org/ 10.1016/ S1469-0292(00)00015-7

Hausenblas, H. A., \& D owns, D. S. (2002b). How Much isToo Much?T he Development and Validation of the ExerciseD ependence Scale. Psychology \& H ealth, 17(4), 387-404. https:// doi.org/10.1080/ 0887044022000004894

Kline, R. B. (1998). Principles and practice of structural equation modeling. Guilford Press.

Lichtenstein, M. B., Hinze, C. J. , Emborg, B., Thomsen, F. \& Hemmingsen, S. D. (2017). Compulsive exercise: links, risks and challenges faced. Psychology research and behavior management, 10, 85-95. https:/ / doi.org/ 10.2147/ PRBM.S113093

Lindwall, M., \& Palmeira, A. (2009). Factorial Validity and Invariance Testing of the Exercise Dependence Scale-Revised in Swedish and Portuguese Exercisers. $M$ easurement in Physical Education and Exercise Science, 13(3), 166-179. https:/ / doi.org/ 10.1080/ 10913670903050313

O gden, J., Veale, D., \& Summers, Z. (1997). The development and validation of the Exercise Dependence Q uestionnaire. Addiction Research, 5(4), 343-355. https:// doi.org/10.3109/ 16066359709004348

Pasman, L. N., \& Thompson, J. K. (1988). Body image and eating disturbance in obligatory runners, obligatory weightlifters, and sedentary individuals.
International Journal of Eating Disorders, 7(6), 759- 769.

Pujals, C., Baile J. I. \& \& González-Calderón, M. J. (2018).

Evaluación de las propiedades psicométricas de la

Escala Revisada de Dependencia del Ejercicio (EDS-

$R$ ) en una muestra española. Pensando Psicología, 14(23). https:/ / doi.org/ 10.16925/ pe.v14i23.2267

Ruiz-Juan, F., \& Zarauz, A. (2011). Validation of the Spanish version of the Commitment to Running Scale (CR). Estudios de Psicología, 32 (2), 195-207. https:/ / doi.org/ 10.1174/ 021093911795978199

Sancho, A., Ruiz-Juan, F., \& Arufe Giraldez, V. (2018). Adicción al atletismo en veteranos: un estudio con variables psicológicas y hábitos de entrenamiento. Retos, 35, 201-207. https:/ / doi. org/ 10.47197/ retos. v0i35. 67472

Sicilia, Á., \& González-Cutre, D. (2011). Dependence and Physical Exercise: Spanish Validation of the Exercise Dependence Scale-Revised (EDS-R). The Spanish Journal of Psychology, 14(01), 421-431. https:/ / doi.org/ 10.5209/ rev sjop.2011.v14.n1.38

Sicilia, Á. , Alías-García, A., Ferriz, R., \& M oreno-M urcia, J. A. (2013). Spanish adaptation and validation of the Exercise Addiction Inventory (EAI). Psicothema, 25(3), 377-383. https:/ / doi. org/ 10.7334/ psicothema2013.21

Simón-Grima, J., San Martín-Salvador, A., EstradaMarcén, N., \& Casterad-Seral, J. (2020). Relación entre la adicción al ejercicio, el uso de dispositivos fitness y la ansiedad rasgo (Relationship between exercise addiction, use of fitness devices and trait anxiety). Retos, 39, 525-531. https:/ / doi.org/ $10.47197 /$ retos. V0i39.80078

Terry, A., Szabo, A . \& G riffiths, M. (2004). The exercise addiction inventory: a new brief screening tool. Addiction Research andTheory, 12(5), 489 - 499. https:/ / doi.org/ 10.1080/ 16066350310001637363

Zarauz Sancho,A., \& Ruiz-Juan, F. (2011). Psychometric properties of the Spanish version of the Running Addiction Scale (RAS). The Spanish Journal of Psychology, 14(2), 967-976. https:/ / doi. org/ 10.5209/ rev_sjop.2011.v14.n2.42

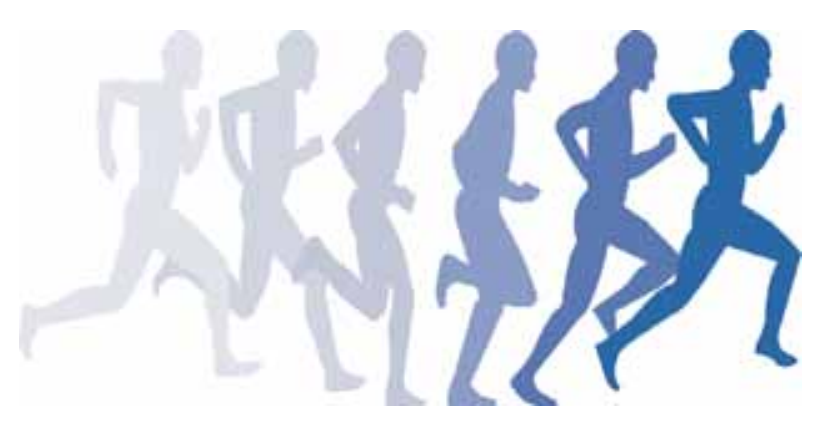




\title{
Propiedades psicométricas de la Escala de Dependencia al Ejercicio-Revisada (EDS-R) en usuarios colombianos de gimnasios Psychometric properties of the Exercise Dependence Scale -Revised (EDS-R) in Colombian gyms users \\ * Jorge Emiro Restrepo, **Tatiana Castañeda Q uirama, ***Gina Paula Cuartas M ontoya \\ *Tecnológico deAntioquia(Colombia), **Politécnico Grancolombiano (Colombia) ***U niversidad Cooperativa de Colombia
} (Colombia)

\begin{abstract}
Resumen: el artículo presentaun estudio psicométrico de laEscala de D ependenciaal Ejercicio Físico - Revisada (EDS-R) en usuariosde gimnasios colombianos con el objetivo de analizar su estructura factorial, consistencia interna, confiabilidad de constructo y su validez convergente, discriminantey de criterio. Se realizó unainvestigación instrumental en laqueparticiparon 515 usuarios degimnasios (44.3\% hombresy $55.7 \%$ mujeres) con edadesentre los 18 y los 58 años ( $M d=25 ; R I Q=21-30)$ de laciudad de M edellín. Se analizó laversión en español (Sicilia\& González-Cutre, 2011) de laEscala de D ependenciaal Ejercicio Físico - Revisada (EDS-R) (D owns, Hausenblas \& Nigg, 2004). Se ejecutaron análisisfactoriales confirmatorios y exploratorios. Resultados: laestructuraoriginal de laEDS-R mostró una adecuada consistenciainternay validez factorial, confirmadaatravés de losíndices de bondad deajuste parael modelo de siete dimensiones. El alfa de Cronbach fue de .917 y el coeficiente de dos mitades de Guttman fuede.924. Losíndices de bondad de ajuste paralaversión original fueron: PCM IN $/$ DF $=2.291 ; \mathrm{RMR}=.091 ; \mathrm{GFI}=.917 ; \mathrm{AGFI}=.886 ; \mathrm{CFI}=.951 ; \mathrm{RMSEA}=.056(90 \% \mathrm{Cl}=.049-.063) ; \mathrm{PCLOSE}=.093$. Sin embargo, losvaloresde lavarianzamediaextraída, lamáximavarianza compartiday lafiabilidad máxima pusieron en evidenciadificultades con la confiabilidad de constructo, la validez convergente y validez discriminante. Tampoco hubo buenos resultados al anal izar la val idez de criterio. Para analizar si los datos en la muestra se ajustaban mejor a una estructura factorial diferente, se realizó un análisis factorial exploratorio complementario que llegó a un modelo de cinco factores, con los mismos 21 ítems. Sin embargo, laeval uación dela fiabilidad compuesta, validez convergentey validez discriminante arrojó mejores resultadosqueel modelo de sietefactores.
\end{abstract}

Palabrasclave: validez, confiabilidad, medición, dependenciaal ejercicio físico.

\begin{abstract}
The article presents apsychometric study of the Physical ExerciseD ependence Scale-Revised (EDS-R) in Colombian gym users with the aim of analyzing itsfactorial structure, inter nal consistency, construct reliability and its convergent, discriminant and criterion validity. An instrumental research was carried out with the participation of 515 gym users (44.3\% men and $55.7 \%$ women) aged between 18 and 58 years ( $M d=25 ; R I Q=21-30$ ) from the city of M edellín. The Spanish version (Sicilia\& G onzález-Cutre, 2011) of the Physical Exercise DependenceScale-Revised (EDS-R) (D owns, H ausenblas\& Nigg, 2004) was analyzed. Confirmatory and exploratory factor analyses were performed. Results: The original structure of theEDS-R showed adequate internal consistency and factorial validity, confirmed through the goodness-of-fit indices for the seven-dimensional model. Cronbach's al phaw was. 917 and the Guttman two-half coefficient was .924. The goodness-of-fit indicesfor the original version were: $\mathrm{PCM} I N / \mathrm{DF}=2.291 ; \mathrm{RMR}=.091 ; \mathrm{GFI}=.917 ; \mathrm{AGFI}=.886 ; \mathrm{CFI}=.951 ; \mathrm{RMSEA}$ $=.056(90 \% \mathrm{Cl}=.049-.063) ; \mathrm{PCLOSE}=.093$. However, the valuesfor mean variance extracted, maximum shared variance and maximum reliability reveal ed difficulties with construct reliability, convergent validity and discriminant validity. There wereal so no-good results when analyzing criterion vali idity. To analyze whether the data in the sample fit better to adifferent factor structure, a complementary exploratory factor analysis was performed that arrived at afive-factor model, with the same 21 items. However, the assessment of composite reliability, convergent validity and discriminant validity yielded better resultsthan the seven-factor model.
\end{abstract}

Key words. validity, reliability, measurement, dependence on physical exercise.

\section{Introducción}

Las adicciones conductuales son un campo de estudios en desar rollo. En la actual idad, solo el juego patológico está incluido en el DSM-5 como un trastorno no relacionado con sustancias dentro de la categoría deTrastornos relacionados con sustancias y trastornos adictivos. Aunque no están incluidos en esta clasificación, diversos comportamientos compulsivos tienen características

Fecha recepción: 23-11-20. Fecha de aceptación: 18-02-21 Jorge Emiro Restrepo jorge. restrep067@ tdea.edu.co psicopatológicas muy similares a las de las adicciones a sustancias, como la adicción a internet, la adicción al sexo, la adicción a los video juegos, entre otras (Grant, Potenza, Weinstein \& Gorelick, 2010).

Practicar ejercicio frecuentemente es en la actualidad, y en la cultura occidental, un comportamiento común. En condiciones normales, es una actividad saluda ble y se llegó a considerar como una adicción positiva (Glasser, 1976). Sin embargo, en al gunos casosesta práctica puede llegar a ser excesiva y descontrolada y puede, incluso, desencadenar síntomas comportamentales, cognitivos y emocionales propios de una adicción tal y como está conceptualizada en el DSM-5. Cuando esto 